\title{
Quantum quench from a thermal tensor state: boundary effects and generalized Gibbs ensemble
}

\author{
Mario Collura ${ }^{1}$ and Dragi Karevski ${ }^{2}$ \\ ${ }^{1}$ Dipartimento di Fisica dell'Università di Pisa and INFN, 56127 Pisa, Italy \\ ${ }^{2}$ Institut Jean Lamour, dpt. P2M, Groupe de Physique Statistique, \\ Université de Lorraine, CNRS, B.P. 70239, F-54506 Vandoeuvre-les-Nancy Cedex, France
}

(Dated: March 15, 2021)

\begin{abstract}
We consider a quantum quench in a non-interacting fermionic one-dimensional field-theory. The system of size $L$ is initially prepared into two halves $\mathcal{L}([-L / 2,0])$ and $\mathcal{R}([0, L / 2])$, each of them thermalized at two different temperatures, $T_{\mathcal{L}}$ and $T_{\mathcal{R}}$ respectively. At a given time the two halves are joined together by a local coupling and the whole system is left to evolve unitarily. For an infinitely extended system $(L \rightarrow \infty)$, we show that the time evolution of the particle and energy densities is well described via a hydrodynamic approach which allows us to evaluate the correspondent stationary currents. We show, in such a case, that the two-point correlation functions are deduced, at large times, from a simple non-equilibrium steady state. Otherwise, whenever the boundary conditions are retained (in a properly defined thermodynamic limit), any current is suppressed at large times, and the stationary state is described by a generalized Gibbs ensemble, which is diagonal and depends only on the post-quench mode occupation.
\end{abstract}

\section{INTRODUCTION}

In the last few years there was a surprisingly growth on the theoretical study of the non-equilibrium properties of many-body quantum systems. This is chiefly due to the enhancement of the experimental techniques which allow to manipulate with great precision trapped ultracold atomic gases, without any significant coupling with the environment ${ }^{1-11}$. Among all, of remarkable interest is the unitary dynamics of a quantum system initially prepared in a non-equilibrium state. In particular, an extremely important question is how to characterize the stationary state to which the system should relax. Indeed, depending on the integrability of the Hamiltonian governing the time evolution, the stationary value of local observables can be described either by an effective Canonical ensemble or by a generalized Gibbs ensemble (GGE), respectively for non-integrable and integrable systems (see Ref. 12 for a review). Many investigations have confirmed this scenario ${ }^{13-41}$ and some effort have been also done in order to understand the role of the initial state in the construction of the GGE ${ }^{18,33,34}$.

In this respect, a very interesting non-equilibrium situation which is considered is a gas of atoms initially split into two different packets, each of them prepared at given initial temperature. The gas is then released and left to evolve freely. A similar problem has already been addressed, using different analytical approaches, for systems defined on a lattice ${ }^{42-47}$. Nevertheless, those studies were focused on the non-equilibrium stationary state which is reached for an infinitely extended system. Indeed, such a non-equilibrium steady-state can be seen as the state asymptotically realized from the initial state with its boundaries always connected to reservoirs at the initial temperatures ${ }^{48}$. In other words, such infinitely extended system will always have, at each instant of time, two infinite portions (the far left and the far right play- ing the role of reservoirs) that remain equilibrated at the two initial temperatures. Between these two reservoirs, the (finite portion of the) system will show nonlocal properties reflecting the non-local structure of the non-equilibrium steady state (which is widely believed conjecture $)^{49-56}$. Recently, by means of thermodynamic Bethe anstaz ${ }^{57}$, the energy current in non-equilibrium steady states of integrable models of relativistic quantum field theory has been evaluated ${ }^{58}$ and interestingly, seems to be in disagreement with what has been numerically found in Ref. 47 (at least within the numerical precision).

In this paper, we focus our attention on a gas of non-interacting spinless fermions either considering the infinite-size limit or taking into account the effects of the boundaries. After preparing the system in a tensor thermal state, i.e. a tensor product of two different thermal density matrices at two different temperature, we leave it to evolve with a non-interacting fermionic Hamiltonian. In particular, for an infinite system, by means of a hydrodynamic description ${ }^{59-61}$, we fully characterize the time-evolution of the particle density and energy density profiles. From these results, we recover the Conformal Field Theory (CFT) predictions for the energy current flowing throughout the system ${ }^{62}$. Furthermore, we inspect the stationary state characterizing the infinitely extended system which does not agree with a local GGE description: indeed, the local conserved charges appearing in the description of the non-equilibrium stationary state combines in such a way that the resulting "effective" Hamiltonian is long-range interacting ${ }^{43}$. In this respect, it has been shown that such a non-locality may drastically affect the mutual information between two adjacent subsystems ${ }^{63}$.

Notwithstanding, locality in the GGE is fully recovered whenever one preserves the effects of the boundaries. We propose a genuine physical interpretation: the traveling particles, confined in a finite region, can reach the edges 
and, being reflected at different times, cause a global dephasing giving rise to a true equilibrium state (no more currents) that is described by the GGE. In other words, we show that, in a properly defined thermodynamic (TD) limit, the reduced density matrix of any finite subsystem converges for long times to the GGE one. This implies that any measurable local observable will converge to the GGE predictions.

The paper is organized as follows. In Sec. II we present the model and the post-quench Hamiltonian which governs the unitary evolution. In Sec. III we characterize the initial thermal tensor state. In Sec. IV we study the time evolution of the two-point correlation function focusing our attention on the particle and energy density profiles. We introduce a hydrodynamic approach describing those quantities and we derive the corresponding particle and energy currents. Finally, we also discuss the approach to the stationary values and we compare the two different scaling regime (with or without boundaries) which lead to different asymptotic results. In Sec. $\mathrm{V}$ we finally show the structure of the GGE and we stress that all local observables are described by the GGE in the fermionic momentum occupation numbers provided that the scaling limit is taken by considering the effects of the boundaries. Finally in Sec. VI we draw our conclusions.

\section{THE MODEL AND THE POST-QUENCH HAMILTONIAN}

We consider a non-relativistic quantum-field theory describing non-interacting spinless fermions in one spatial dimension. The system is defined in the symmetric interval $[-L / 2, L / 2]$ with open boundary conditions (OBC), and where we introduced the system length $L$ in order to regularize infrared divergencies. At the end, we will be interested in the thermodynamic limit (TD limit) $L \rightarrow \infty$. The Hamiltonian governing the unitary evolution of the system is given by

$$
\hat{H}=\int_{-L / 2}^{L / 2} d x \partial_{x} \hat{\Psi}^{\dagger}(x) \partial_{x} \hat{\Psi}(x)
$$

where the fields $\hat{\Psi}(x), \hat{\Psi}^{\dagger}(x)$ satisfy the canonical anticommutation rules $\left\{\hat{\Psi}(x), \hat{\Psi}^{\dagger}(y)\right\}=\delta(x-y)$. Introducing the normal free-fermionic operators

$$
\begin{aligned}
\hat{\eta}_{p_{n}} & =\int_{-L / 2}^{L / 2} d x \varphi_{p_{n}}(x) \hat{\Psi}(x) \\
\varphi_{p_{n}}(x) & =\sqrt{\frac{2}{L}} \sin \left[p_{n}\left(x+\frac{L}{2}\right)\right], p_{n}=\frac{\pi}{L} n
\end{aligned}
$$

with $n \in \mathbb{N}$, the Hamiltonian (1) is readily diagonalized

$$
\hat{H}=\sum_{n=0}^{\infty} p_{n}^{2} \hat{n}_{p_{n}}
$$

with $\hat{n}_{p_{n}} \equiv \hat{\eta}_{p_{n}}^{\dagger} \hat{\eta}_{p_{n}}$ the fermionic mode occupation operator. Notice that the Hamiltonian commutes with the total number of particles operator

$$
\hat{N}=\int_{-L / 2}^{L / 2} d x \hat{\Psi}^{\dagger}(x) \hat{\Psi}(x)=\sum_{n=0}^{\infty} \hat{n}_{p_{n}},
$$

therefore, $\hat{H}$ and $\hat{N}$ can be simultaneously diagonalized in the many-body Hilbert space. Since the excitation spectrum of the Hamiltonian is non-negative, the ground state without fixing the number of particles is the vacuum state $|0\rangle$, such that $\hat{\eta}_{p_{n}}|0\rangle=0, \forall n>0$.

\section{THE INITIAL SYSTEM}

At time $t=0$ the system is divided into two halves $\mathcal{L}$ $(x<0)$ and $\mathcal{R}(x>0)$. Therefore the two subsystems are initially uncorrelated and the total initial Hamiltonian is the direct sum of two independent Hamiltonians which can be diagonalized separately. Indeed, the Hamiltonian describing a subsystem is given by Eq.(1) where the integration domain is reduced to a half interval: $[0, L / 2]$ or $[-L / 2,0]$ for the right and left subsystems respectively.

The normalized eigenfunctions, building-up the oneparticle Hilbert space of the semi-intervals, are given by

$$
\phi_{q_{m}}^{ \pm}(x)=\frac{2}{\sqrt{L}} \sin \left(q_{m} x\right) \theta( \pm x), q_{m}=\frac{2 \pi}{L} m, m \in \mathbb{N},
$$

where $\theta(x)$ is the Heaviside function such that $\theta(x)=1$ for $x>0$, and zero otherwise. Thanks to these functions the half-interval Hamiltonians are straightforwardly diagonalized. For example, in the sub-interval $[0, L / 2]$ one has

$$
\hat{H}_{0}^{+}=\int_{0}^{L / 2} d x \partial_{x} \hat{\Psi}^{\dagger}(x) \partial_{x} \hat{\Psi}(x)=\sum_{m=0}^{\infty} q_{m}^{2} \hat{\xi}_{q_{m}}^{\dagger} \hat{\xi}_{q_{m}}
$$

where the fermionic fields $\Psi, \Psi^{\dagger}$ are related to the diagonal operators via

$$
\hat{\Psi}(x)=\sum_{m=0}^{\infty} \phi_{q_{m}}^{+}(x) \hat{\xi}_{q_{m}}, \quad x>0 .
$$

Similar arguments are valid for the negative half-interval.

\section{A. The overlap}

It is useful to express the initial one-particle eigenfunctions $\phi_{q_{m}}^{ \pm}(x)$ in terms of the post-quench one-particle eigenfunctions $\varphi_{p_{n}}(x)$, i.e.

$$
\phi_{q_{m}}^{ \pm}(x)=\sum_{n=0}^{\infty} A_{n, m}^{ \pm} \varphi_{p_{n}}(x)
$$



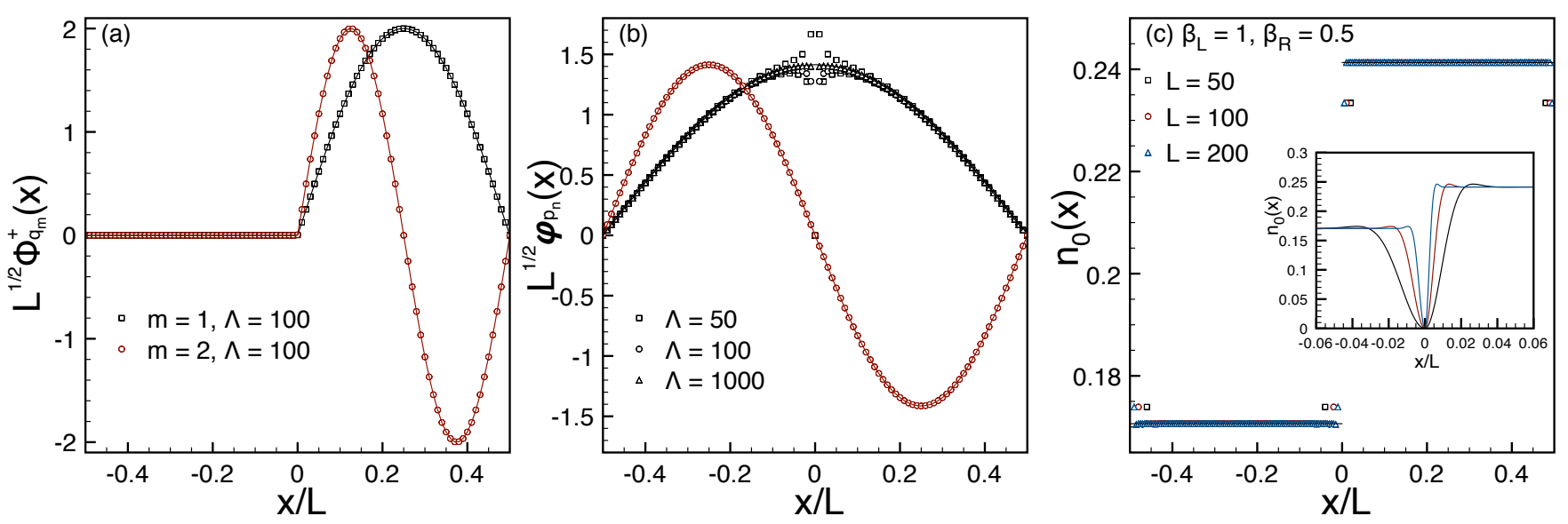

Figure 1: (a) Pre-quench eigenfunctions $\phi_{q_{m}}^{+}(x)$ (full lines) compared with the series in Eq.(9) truncated at a given $\Lambda$ for the first two level $m=1,2$ (symbols). (b) The post-quench eigenfunctions $\varphi_{p_{n}}(x)$ (full lines) for $n=1,2$ compared with the series in Eq.(11) truncated at a given $\Lambda$ (symbols). Notice how, for $n=1$ the sum approaches the eigenfunction apart from $x=0$, wherein the series (11) is identically zero. (c) Initial density profile for a system prepared with $\beta_{\mathcal{L}}=1$ and $\beta_{\mathcal{R}}=0.5$, and different sizes $L$. In the inset we zoomed the region close to the origin; notice how, the singular behavior in $x=0$ affects a region around the origin which shrinks as the system size $L$ increases.

where we have explicitly introduced the overlap

$$
A_{n, m}^{ \pm}=\int_{-L / 2}^{L / 2} d x \varphi_{p_{n}}(x) \phi_{q_{m}}^{ \pm}(x) .
$$

Notice that Eq. (9) is exact since the post-quench oneparticle eigenstates are a complete basis in the symmetric interval $[-L / 2, L / 2]$. Otherwise, in order to invert such a relation, and to rewrite $\varphi_{p_{n}}(x)$ in terms of the prequench eigenstates, one needs a linear combination of both $\phi_{q_{m}}^{-}(x)$ and $\phi_{q_{m}}^{+}(x)$,

$$
\varphi_{p_{n}}(x)=\sum_{m=0}^{\infty}\left[A_{n, m}^{-} \phi_{q_{m}}^{-}(x)+A_{n, m}^{+} \phi_{q_{m}}^{+}(x)\right] .
$$

Nevertheless, for $n$ odd, the series in Eq.(11) is not absolutely convergent in $[-L / 2, L / 2]$ due to the anomalous behavior in $x=0$. However, such anomaly can be neglected in the thermodynamic limit since $x=0$ is a subset of measure zero in $\mathbb{R}$. The overlap can be explicitly evaluated, giving

$$
A_{n, m}^{ \pm}=\left\{\begin{array}{cl} 
\pm \frac{4 \sqrt{2} m \sin (n \pi / 2)}{\pi\left(4 m^{2}-n^{2}\right)} & \text { for } n \text { odd, } \\
\frac{(-1)^{m}}{\sqrt{2}} \delta_{n, 2 m} & \text { for } n \text { even. }
\end{array}\right.
$$

In Figure 1 we show how the series in Eq.(9) and Eq.(11) reproduce the correct post(pre)-quench oneparticle eigenfunctions.

\section{B. Thermal tensor state}

The initial state is constructed as a tensor product of two thermal density matrices at two different temperatures, i.e. $\hat{\rho}_{0}=\hat{\varrho}_{-}\left(\beta_{\mathcal{L}}\right) \otimes \hat{\varrho}_{+}\left(\beta_{\mathcal{R}}\right)$, where $\hat{\varrho}_{ \pm}(\beta)=$
$Z^{-1} \exp \left(-\beta \hat{H}_{0}^{ \pm}\right)$. This means that the two spatial regions $(\mathcal{L}$ and $\mathcal{R})$ are initially uncorrelated. Furthermore, such a state is quadratic in the local field operators and, therefore, the Wick's theorem applies.

Thanks to this fact, the initial two-point correlation function $C_{0}(x, y) \equiv\left\langle\hat{\Psi}^{\dagger}(x) \hat{\Psi}(y)\right\rangle_{0}$ splits into two separate terms

$$
C_{0}(x, y)=C_{\mathcal{L}}(x, y) \theta(-x) \theta(-y)+C_{\mathcal{R}}(x, y) \theta(x) \theta(y)
$$

with

$$
\begin{aligned}
C_{\mathcal{L} / \mathcal{R}}(x, y) & =\frac{4}{L} \sum_{m=0}^{\infty} \frac{\sin \left(q_{m} x\right) \sin \left(q_{m} y\right)}{1+\exp \left(\beta_{\mathcal{L} / \mathcal{R}} q_{m}^{2}\right)} \\
& =\frac{2}{L} \sum_{m=0}^{\infty} \frac{\cos \left[q_{m}(x-y)\right]}{1+\exp \left(\beta_{\mathcal{L} / \mathcal{R}} q_{m}^{2}\right)} \\
& -\frac{2}{L} \sum_{m=0}^{\infty} \frac{\cos \left[q_{m}(x+y)\right]}{1+\exp \left(\beta_{\mathcal{L} / \mathcal{R}} q_{m}^{2}\right)}
\end{aligned}
$$

Notice that the initial correlation function depends both on $x-y$ and $x+y$ due to the fact that the initial state breaks the translational invariance, even in the thermodynamic limit. Indeed, taking the TD limit, thanks to the parity of the fermionic distribution, we can rewrite the latter as

$$
\begin{aligned}
C_{\mathcal{L} / \mathcal{R}}(x, y) & =\beta_{\mathcal{L} / \mathcal{R}}^{-1 / 2} \mathcal{F}\left[(x-y) \beta_{\mathcal{L} / \mathcal{R}}^{-1 / 2}\right] \\
& -\beta_{\mathcal{L} / \mathcal{R}}^{-1 / 2} \mathcal{F}\left[(x+y) \beta_{\mathcal{L} / \mathcal{R}}^{-1 / 2}\right]
\end{aligned}
$$

where we introduced the Fourier transform

$$
\mathcal{F}(z)=\int_{-\infty}^{\infty} \frac{d q}{2 \pi} \frac{\exp (i z q)}{1+\exp \left(q^{2}\right)}
$$



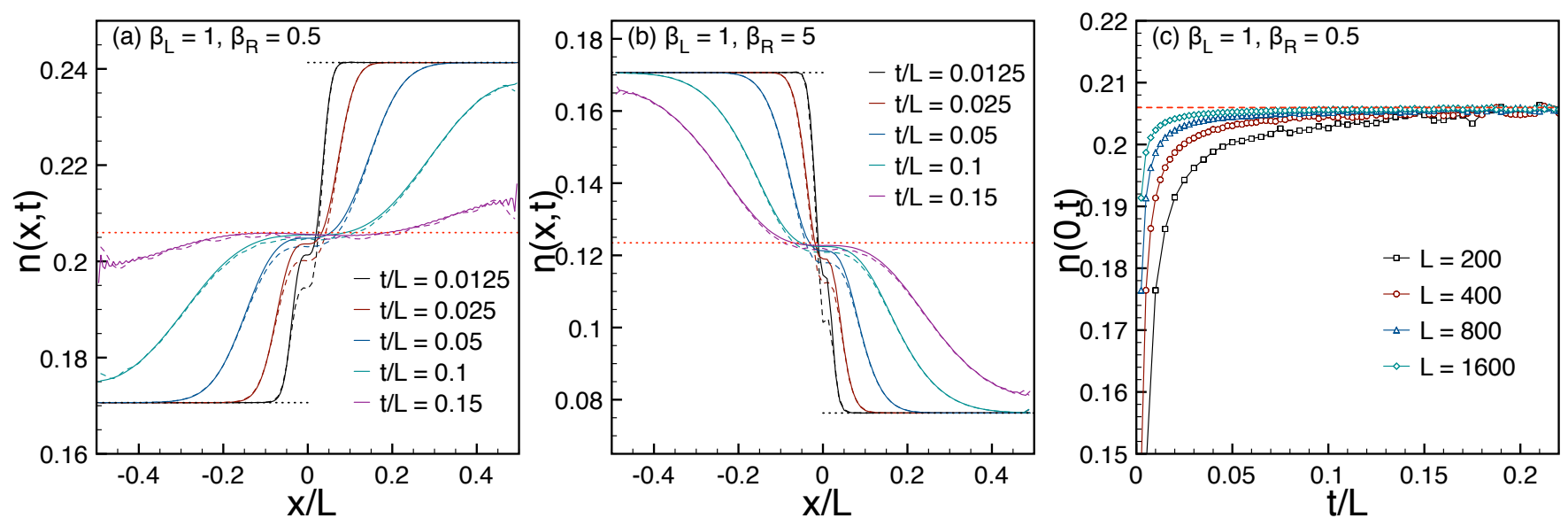

Figure 2: Particle density profiles vs rescaled space $x / L$ at different rescaled times $t / L$ for a system initially prepared with $\beta_{\mathcal{L}}=1$ and $\beta_{\mathcal{R}}=0.5$ (a) and $\beta_{\mathcal{R}}=5$ (b). Dashed lines correspond to $L=400$, full lines to $L=1000$. Dotted lines represent the initial densities (black dotted) and the stationary density (red dotted) in the TD limit. (c) Evolution of the local particle density at $x=0$ for different sizes $L$. Notice how, in terms of the rescaled time $t / L$, the curves approach a step-like function as $L$ increases.

Using the Taylor's series expansion $(1+z)^{-1}=$ $-\sum_{n=1}^{\infty}(-1)^{n} z^{-n}$, we have

$$
\mathcal{F}(z)=-\frac{1}{2 \sqrt{\pi}} \sum_{n=1}^{\infty} \frac{(-1)^{n} \exp \left[-z^{2} /(4 n)\right]}{\sqrt{n}} .
$$

Integrating Eq.(14) with $y=x$, and using the orthogonality of the one-particle eigenfunctions, one immediately obtains the left (right) number of particles

$$
\begin{aligned}
N_{\mathcal{L} / \mathcal{R}} & =\sum_{m=0}^{\infty} \frac{1}{1+\exp \left(\beta_{\mathcal{L} / \mathcal{R}} q_{m}^{2}\right)} \\
& =\frac{L}{\sqrt{\beta_{\mathcal{L} / \mathcal{R}}}} \int_{0}^{\infty} \frac{d q}{2 \pi} \frac{1}{1+\exp \left(q^{2}\right)} \\
& =L \frac{(1-\sqrt{2}) \zeta(1 / 2)}{4 \sqrt{\pi} \sqrt{\beta_{\mathcal{L} / \mathcal{R}}}}
\end{aligned}
$$

where $\zeta(z)=\sum_{k=1}^{\infty} k^{-z}$ is the Reimann zeta function. Notice that the number of particle is, as it should, diverging in the TD limit making finite the initial left and right densities $n_{\mathcal{L} / \mathcal{R}} \equiv 2 N_{\mathcal{L} / \mathcal{R}} / L$. Moreover, in the TD limit the left and right initial densities are uniform. In Figure 1 we plot the numerical evaluated initial densities for different system sizes.

In the same way, we can evaluate the left (right) total energy in the TD limit. Indeed, from Eq.(7) one immediately has

$$
\begin{aligned}
E_{\mathcal{L} / \mathcal{R}} & =\sum_{m=0}^{\infty} \frac{q_{m}^{2}}{1+\exp \left(\beta_{\mathcal{L} / \mathcal{R}} q_{m}^{2}\right)} \\
& =\frac{L}{\beta_{\mathcal{L} / \mathcal{R}}^{3 / 2}} \int_{0}^{\infty} \frac{d q}{2 \pi} \frac{q^{2}}{1+\exp \left(q^{2}\right)} \\
& =L \frac{(2-\sqrt{2}) \zeta(3 / 2)}{16 \sqrt{\pi} \beta_{\mathcal{L} / \mathcal{R}}^{3 / 2}}
\end{aligned}
$$

which gives rise to finite energy densities $\mathcal{E}_{\mathcal{L} / \mathcal{R}} \equiv$ $2 E_{\mathcal{L} / \mathcal{R}} / L$.

\section{THE QUENCH PROTOCOL}

In this section we analyze the out-off-equilibrium dynamics after putting in contact the two halves $\mathcal{L}$ and $\mathcal{R}$. In other words, the initial state $\hat{\rho}_{0}$ evolves unitarily according to the post-quench Hamiltonian $\hat{H}$. In particular, since the Hamiltonian is quadratic in the fermionic operators, the evolved state keeps its gaussian character and the Wick's theorem still applies. Consequently, all observables are derived from the two-point correlation function. Therefore, we focus our attention on the timeevolution of the two-point correlation function which is given by

$$
C(x, y ; t)=\sum_{n, m=0}^{\infty} \varphi_{p_{n}}(x) \varphi_{p_{m}}(y)\left\langle\hat{\eta}_{p_{n}}^{\dagger} \hat{\eta}_{p_{m}}\right\rangle_{t},
$$

where the evolution of the diagonal operators is trivially given by

$$
\left\langle\hat{\eta}_{p_{n}}^{\dagger} \hat{\eta}_{p_{m}}\right\rangle_{t}=\exp \left[i\left(p_{n}^{2}-p_{m}^{2}\right) t\right]\left\langle\hat{\eta}_{p_{n}}^{\dagger} \hat{\eta}_{p_{m}}\right\rangle_{0}
$$

Using the inverse transformation of Eq. (2), the initial correlation function of the post-quench fermionic operators can be rewritten in terms of $C_{0}(x, y)$

$$
\left\langle\hat{\eta}_{p_{n}}^{\dagger} \hat{\eta}_{p_{m}}\right\rangle_{0}=\iint d z d w \varphi_{p_{n}}(z) \varphi_{p_{m}}(w) C_{0}(z, w) .
$$

Using the overlap between the pre-quench and the postquench one-particle eigenfunctions one obtains

$$
\left\langle\hat{\eta}_{p_{n}}^{\dagger} \hat{\eta}_{p_{m}}\right\rangle_{0}=\sum_{l=0}^{\infty}\left[\frac{A_{n, l}^{-} A_{m, l}^{-}}{1+\exp \left(\beta_{\mathcal{L}} q_{l}^{2}\right)}+\frac{A_{n, l}^{+} A_{m, l}^{+}}{1+\exp \left(\beta_{\mathcal{R}} q_{l}^{2}\right)}\right] .
$$



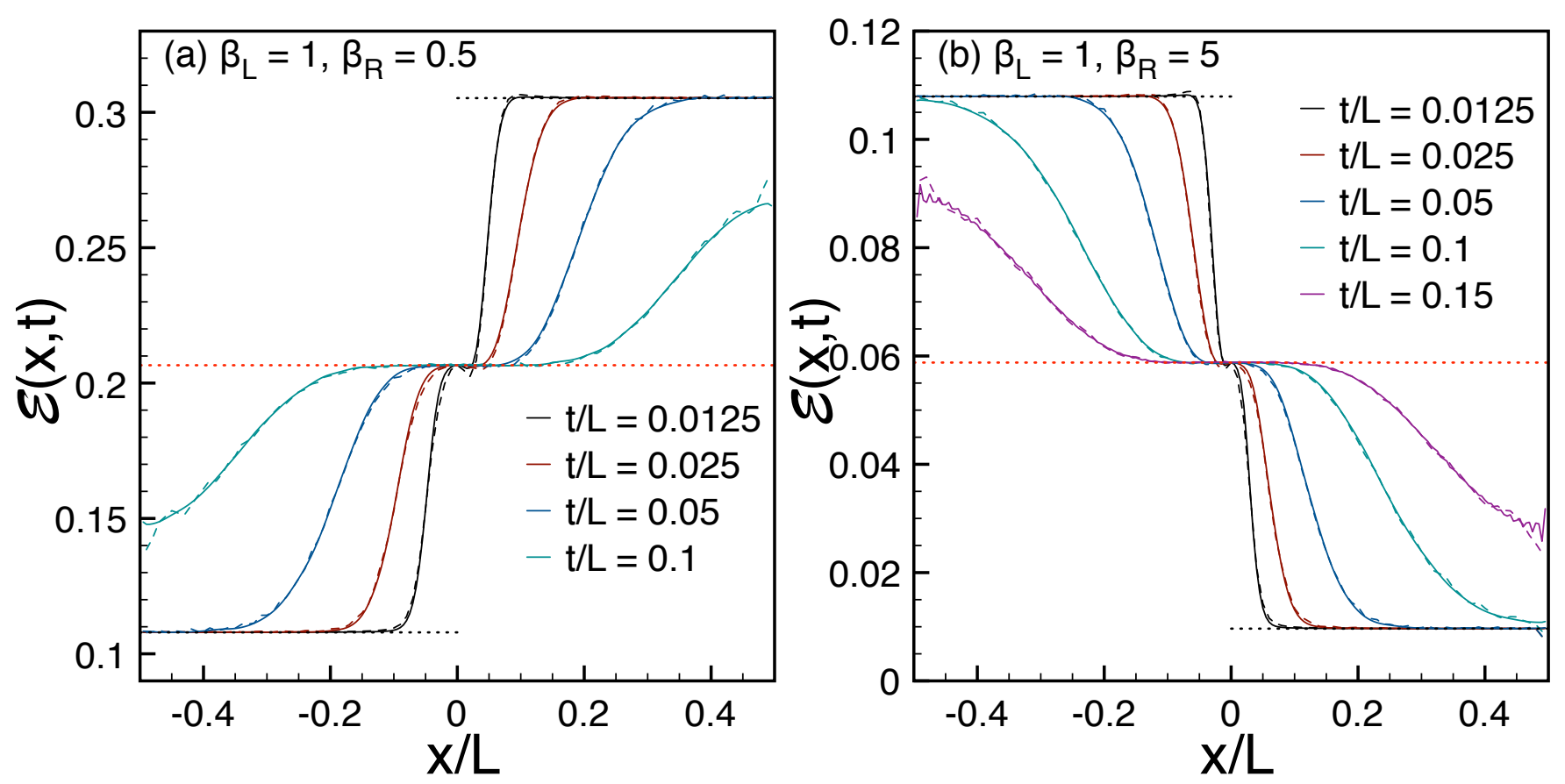

Figure 3: Energy density profiles vs rescaled space $x / L$ at different rescaled times $t / L$ for a system initially prepared with $\beta_{\mathcal{L}}=1$ and $\beta_{\mathcal{R}}=0.5$ (a) and $\beta_{\mathcal{R}}=5$ (b). Dashed lines correspond to $L=400$, full lines to $L=1000$. Dotted lines represent the initial densities (black dotted) and the stationary density (red dotted) in the TD limit.

Finally, using the latter equation, we can rewrite the time evolved correlation function as

$C(x, y ; t)=\sum_{l=0}^{\infty}\left[\frac{\phi_{q_{l}}^{-}(x, t)^{*} \phi_{q_{l}}^{-}(y, t)}{1+\exp \left(\beta_{\mathcal{L}} q_{l}^{2}\right)}+\frac{\phi_{q_{l}}^{+}(x, t)^{*} \phi_{q_{l}}^{+}(y, t)}{1+\exp \left(\beta_{\mathcal{R}} q_{l}^{2}\right)}\right]$

where we introduced the time-evolved one particle eigenfunctions

$$
\phi_{q_{m}}^{ \pm}(x, t)=\sum_{n=0}^{\infty} A_{n, m}^{ \pm} \varphi_{p_{n}}(x) \mathrm{e}^{-i p_{n}^{2} t} .
$$

We numerically evaluated the time-dependent particle density $n(x, t) \equiv C(x, x ; t)$ and we report in Figure 2 the profile at different times for a system of sizes $L=400,1000$. We can see how the density equilibrate toward the stationary value: a causality zone, with homogeneous density, propagates starting from the origin and determines, at each instant of time, an equilibration region $\left[-x^{*}(t), x^{*}(t)\right]$. Notice, however that, for finite systems, the singular behavior in $x=0$ affects the value of the density in such region, which displays size effects. However, whenever the TD limit is considered, the plateau in the density profile around $x=0$ takes a constant value converging as the size is increased to the large-time stationary value (see Figure 2 ).

Furthermore, we also numerically evaluated the energy density profile $\mathcal{E}(x, t) \equiv\left\langle\partial_{x} \hat{\Psi}^{\dagger}(x) \partial_{x} \hat{\Psi}(x)\right\rangle_{t}$ which can be easily shown to be equal to $\left.\partial_{x y}^{2} C(x, y ; t)\right|_{y=x}$. In Figure 3 we plot the energy profiles for different system sizes and different initial temperatures. The same considerations that was done for the particle density applies also in this case. Nevertheless, in this case, thanks to the fact that the energy density depends on the derivative of the oneparticle eigenfunctions, the anomalous behavior in the neighborhood of $x=0$ (which is evident in the particle density) is smoothed out.

\section{A. Hydrodynamic description of the time-dependent profile of local observables}

Taking into account all the considerations of the previous paragraphs, we argue that the dynamics of the particle-density profile as well as of the energy-density profile can be fully characterized, in the TD limit, by a semi-classical description. We want to stress here that such a description, by construction, can not reproduce the dynamics of the correlations. The idea underlying the semi-classical approach was already proposed in Ref. 59-61 and is essentially based on the fact that the quantum dynamics of the local densities is well captured by a hydrodynamic description in the phase-space $(p, x)$ of the correspondent classical coarse-grained densities.

Indeed, if one considers, for example, the particle density, we can associate to each phase-space point a local initial packet of particles $n_{0}(p, x) d p d x$. Then, each of them evolves following a classical trajectory $x^{ \pm}(t)=$ $x_{0} \pm v_{p} t$, with velocity $v_{p}=2 p$, where the sign refers to the left( $(-)$ and right $(+)$ movers. The reason why we have to consider a superposition of two moving packet 

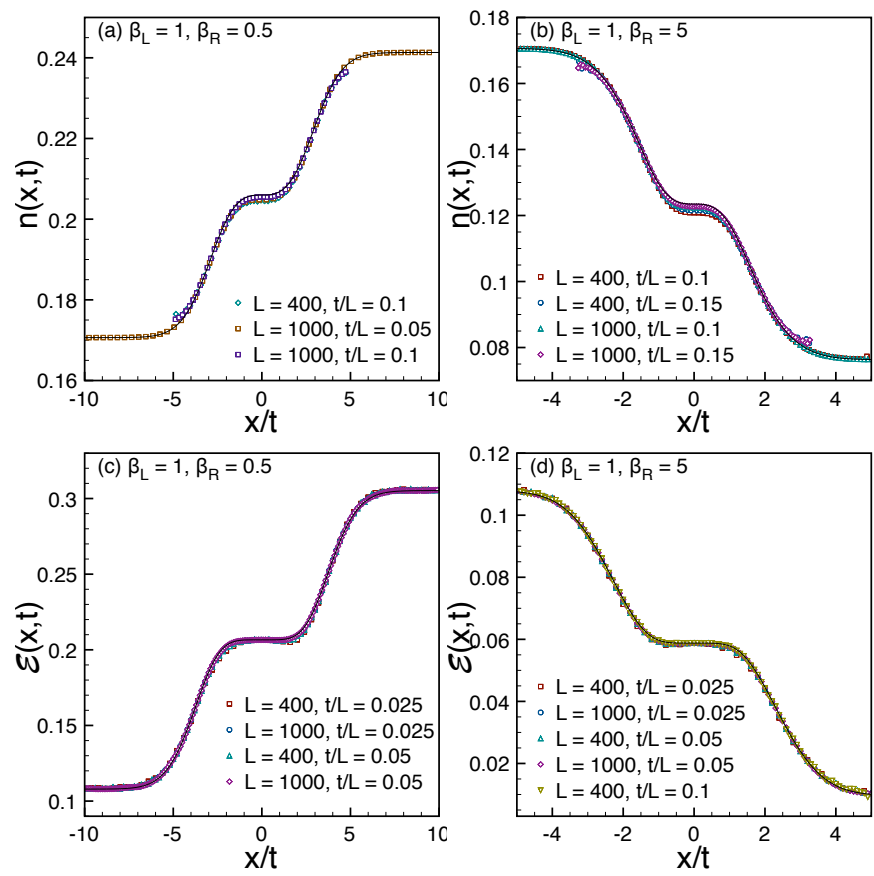

Figure 4: (a,b) Numerically evaluated particle density profile vs the scaling variable $x / t$ for different sizes $L$ and rescaled times $t / L$ (symbols). The full lines are the analytic result (35). (c,d) Numerically evaluated energy density profile vs the scaling variable $x / t$ for different sizes $L$ and rescaled times $t / L$ (symbols). The full lines are the analytic result (38)

is intimately connected to the geometry of the system; indeed we can think at the initial single particle eigenfunctions like a symmetric superposition of propagative modes $\exp ( \pm i p x)$. In our case, the initial particle distribution in the phase-space is

$$
n_{0}(x, p)=\frac{1}{\pi} \frac{\theta(-x)}{1+\exp \left(\beta_{\mathcal{L}} p^{2}\right)}+\frac{1}{\pi} \frac{\theta(x)}{1+\exp \left(\beta_{\mathcal{R}} p^{2}\right)},
$$

from which we can straightforwardly obtain the timeevolved density profile

$$
n(x, t)=\frac{1}{2} \sum_{\sigma= \pm 1} \iint d p d x_{0} n_{0}\left(x_{0}, p\right) \delta\left(x-x_{0}-\sigma 2 p t\right) .
$$

Injecting Eq.(33) in the latter equation, we finally obtain

$$
n(x, t)=\frac{1}{2}\left\{\begin{array}{cc}
n_{\mathcal{R}}+f_{\mathcal{L}}(x / 2 t, \infty)+f_{\mathcal{R}}(0, x / 2 t) & x>0 \\
n_{\mathcal{L}}+f_{\mathcal{L}}(0,-x / 2 t)+f_{\mathcal{R}}(-x / 2 t, \infty) & x<0
\end{array},\right.
$$

where we have introduced the scaling function

$$
f_{\mathcal{L} / \mathcal{R}}(x, y) \equiv \int_{x}^{y} \frac{d p}{\pi} \frac{1}{1+\exp \left(\beta_{\mathcal{L} / \mathcal{R}} p^{2}\right)} .
$$

Following the same lines, we can describe the timedependent energy-density profile. Indeed, starting from the initial energy distribution

$$
\mathcal{E}_{0}(x, p)=\frac{p^{2}}{\pi} \frac{\theta(-x)}{1+\exp \left(\beta_{\mathcal{L}} p^{2}\right)}+\frac{p^{2}}{\pi} \frac{\theta(x)}{1+\exp \left(\beta_{\mathcal{R}} p^{2}\right)},
$$

we straightforwardly obtain

$\mathcal{E}(x, t)=\frac{1}{2}\left\{\begin{array}{cc}\mathcal{E}_{\mathcal{R}}+g_{\mathcal{L}}(x / 2 t, \infty)+g_{\mathcal{R}}(0, x / 2 t) & x>0 \\ \mathcal{E}_{\mathcal{L}}+g_{\mathcal{L}}(0,-x / 2 t)+g_{\mathcal{R}}(-x / 2 t, \infty) & x<0\end{array}\right.$,

with

$$
g_{\mathcal{L} / \mathcal{R}}(x, y) \equiv \int_{x}^{y} \frac{d p}{\pi} \frac{p^{2}}{1+\exp \left(\beta_{\mathcal{L} / \mathcal{R}} p^{2}\right)} .
$$

In Figure 4 we compare the hydrodynamical predictions (35) and (38) with the numerically evaluated particle and energy density profiles for different system sizes $L$ and rescaled times $t / L$ where one can see a perfect agreement.

\section{B. Particle current and energy current}

Using these last results, we can easily derive the particle and the energy currents flowing through the interface separating the two semi-infinite half systems. Indeed, from the continuity equation $\partial_{t} n(x, t)=$ $-\partial_{x} J_{n}(x, t)$ we define the particle current $J_{n}(x, t) \equiv$ $2 \operatorname{Im}\left[\left\langle\hat{\Psi}^{\dagger}(x) \partial_{x} \hat{\Psi}(x)\right\rangle_{t}\right]$. In particular, due to the fact that the current should be vanishing at $x= \pm \infty$, we have

$$
J_{n}(x, t)=-\int_{-\infty}^{x} d z \partial_{t} n(z, t) .
$$

Therefore, using the scaling form (35), the current of particle which flows from $\mathcal{L}$ to $\mathcal{R}$ is given by

$$
\mathcal{J}_{n} \equiv J_{n}(0, t)=\frac{\log (2)}{2 \pi}\left(\frac{1}{\beta_{\mathcal{L}}}-\frac{1}{\beta_{\mathcal{R}}}\right),
$$

which does not depend on time as expected in the scaling regime. Similarly, the energy current $J_{\mathcal{E}}(x, t) \equiv$ $2 \operatorname{Im}\left[\left\langle\partial_{x} \hat{\Psi}^{\dagger}(x) \partial_{x}^{2} \hat{\Psi}(x)\right\rangle_{t}\right]$ is given by

$$
J_{\mathcal{E}}(x, t)=-\int_{-\infty}^{x} d x \partial_{t} \mathcal{E}(x, t),
$$

which evaluated at $x=0$, making use of (38), gives

$$
\mathcal{J}_{\mathcal{E}} \equiv J_{\mathcal{E}}(0, t)=\frac{\pi}{24}\left(\frac{1}{\beta_{\mathcal{L}}^{2}}-\frac{1}{\beta_{\mathcal{R}}^{2}}\right) .
$$

This result agrees with the Conformal Field Theory $(\mathrm{CFT})$ prediction ${ }^{46,62} \mathcal{J}_{\mathcal{E}}^{C F T}=\left[c \pi /\left(12 \hbar v_{F}\right)\right]\left(\beta_{\mathcal{L}}^{-2}-\beta_{\mathcal{R}}^{-2}\right)$, with $\hbar=1$, central charge $c=1$ and Fermi velocity $v_{F}=2$. Interestingly, in Ref. 47 it has been numerically found that, for a large class of problems, the steady-state energy current takes the functional form of a difference between the total radiated power from the left and right leads. However, new recent results on integrable models of relativistic quantum field theory (IQFT) with diagonal scattering seem to show that this property of the current does not hold in general ${ }^{58}$. 


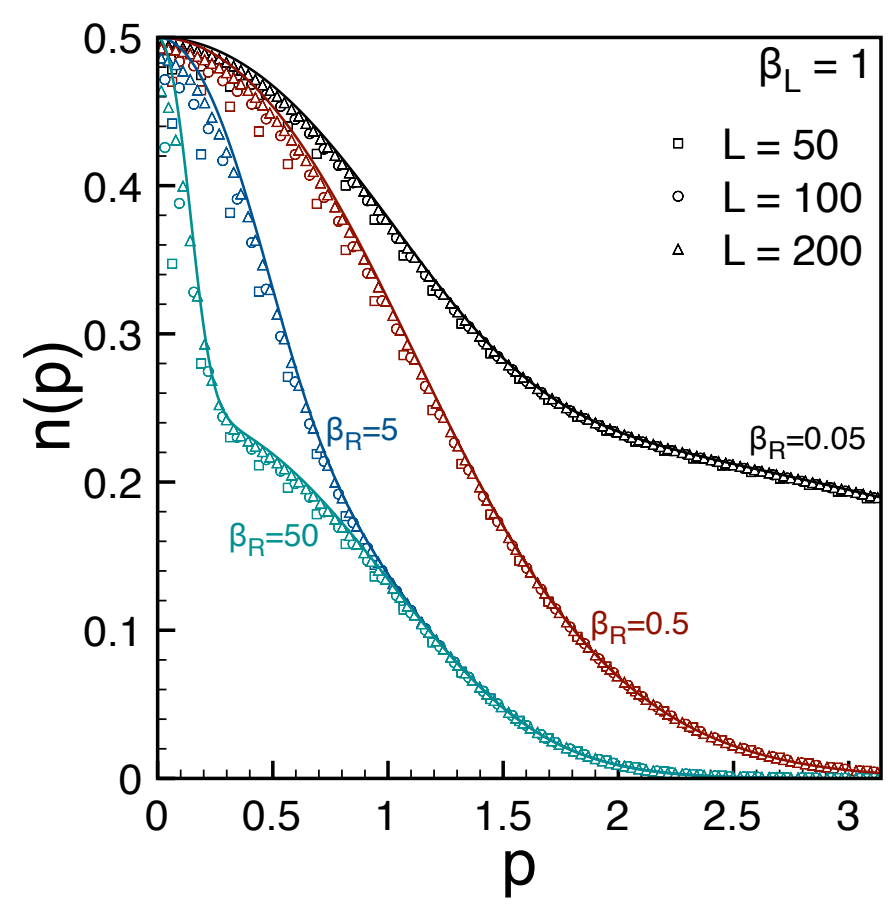

Figure 5: Post-quench mode occupation evaluated for different initial temperatures $\beta_{\mathcal{L}}=1$ and $\beta_{\mathcal{R}}=0.05,0.5,5,50$. Different symbols represent different sizes $L$ showing the convergence toward Eq.(46) (full lines).

\section{Large-time limit and boundary effects}

Non-Equilibrium Steady State (NESS)

The description of the non-equilibrium stationary state in an infinite quantum system was done for the $X Y$ chain in Ref. 42-44 with full mathematical rigor in the framework of $C^{*}$-algebraic dynamical systems. However, in our case, to avoid boundary effects and give a longtime description of the local physics around the connecting point we proceed in the following way: $(i)$ we rewrite the two-point correlation function in the TD limit $(L \rightarrow \infty)$, changing all sums to integrals; (ii) and then take $t \rightarrow \infty$ limit which allows us to use the stationaryphase approximation ${ }^{64,65}$. Following this recipe, the twopoint correlation function in the stationary regime is found to be given by

$$
C^{\text {Stat }}(x, y)=C_{R e}^{\text {Stat }}(x-y)+i C_{I m}^{\text {Stat }}(x-y)
$$

where the real part

$$
C_{R e}^{S t a t}(z)=\int_{0}^{\infty} \frac{d p}{\pi} \cos (p z) n(p)
$$

with

$$
n(p)=\frac{1}{2}\left[\frac{1}{1+\exp \left(\beta_{\mathcal{L}} p^{2}\right)}+\frac{1}{1+\exp \left(\beta_{\mathcal{R}} p^{2}\right)}\right],
$$

and

$$
C_{I m}^{\text {Stat }}(z)=\int_{0}^{\infty} \frac{d p}{2 \pi}\left[\frac{\sin (p z)}{1+\exp \left(\beta_{\mathcal{R}} p^{2}\right)}-\frac{\sin (p z)}{1+\exp \left(\beta_{\mathcal{L}} p^{2}\right)}\right],
$$

for the imaginary part. The $n(p)$ is the TD limit of the initial mode occupation $\left\langle\hat{n}_{p_{n}}\right\rangle_{0}$, i. e. given by the diagonal terms of Eq. (30). In Figure 5, we plot $\left\langle\hat{n}_{p_{n}}\right\rangle_{0}$ for different system sizes, showing the convergence toward the TD-limit result. Introducing the two-sided Fermi distribution $\tilde{f}(p) \equiv \theta(p) \tilde{f}_{\mathcal{L}}(p)+\theta(-p) \tilde{f}_{\mathcal{R}}(p)$, where the momentum is now considered on the whole real axis and with $\tilde{f}_{\mathcal{L} / \mathcal{R}}(p)=\frac{1}{1+e^{\beta} \mathcal{L} / \mathcal{R}^{p^{2}}}$, one can also rewrite the stationary correlation function as the Fourier transform of $\tilde{f}(p)$ :

$$
C^{S t a t}(x, y)=\int_{-\infty}^{\infty} \frac{d p}{2 \pi} \tilde{f}(p) e^{-i p(x-y)}
$$

such that

$$
C_{R e}^{S t a t}(z)=\int_{-\infty}^{\infty} \frac{d p}{2 \pi} \tilde{f}(p) \cos (p z)
$$

and

$$
C_{I m}^{\text {Stat }}(z)=-\int_{-\infty}^{\infty} \frac{d p}{2 \pi} \tilde{f}(p) \sin (p z) .
$$

As a consequence of this redefinition of the momenta $p$, the non-equilibrium stationary state (NESS) described by this correlation function can be viewed as a superposition of left and right stationary movers, each of them being distributed with respect to there own inverse temperature $\beta_{\mathcal{L}}$ and $\beta_{\mathcal{L}}$. In this regime (large time limit and close to the connecting point) translation invariance is restored and the stationary density is uniform over the whole region with $\bar{n}=\left(n_{\mathcal{L}}+n_{\mathcal{R}}\right) / 2$. The imaginary part gives rise to non-vanishing stationary currents (reproducing exactly the results already found in the previous section for $\mathcal{J}_{n}$ and $\mathcal{J}_{\mathcal{E}}$ ) which highlight the nature of the NESS describing the system.

Once again, we want to stress that Eq. (44) which represents the correlation function in the NESS, was found considering an infinitely extended system, i. e. $L \rightarrow \infty$, from the very beginning of the calculation. But of course, the numerical evaluation of the correlation function is done using finite system sizes and as a consequence, the NESS description applies only if $t \ll L$. In Figure 6 we show for two different initial conditions how the timedependent correlation function approaches the stationary value in a region around the origin. Notice that we can not consider large value of $t / L$ if we want to be coherent with the above description of the steady-state.

Equilibration regime

If one extends the numerical evaluation of the postquench correlation function for times such that $t>L$ 

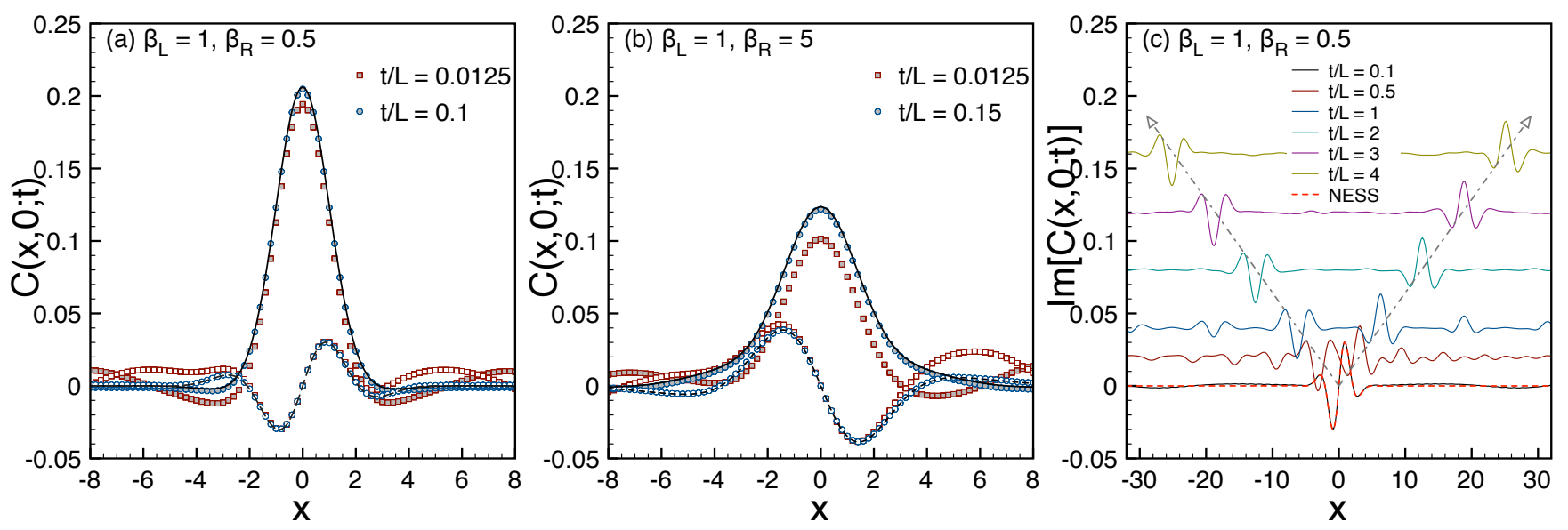

Figure 6: Numerically evaluated correlation function $C(x, 0 ; t)$ for a system with size $L=400$ initially prepared with $\beta_{\mathcal{L}}=1$ and $\beta_{\mathcal{R}}=0.5$ (a) and $\beta_{\mathcal{R}}=5$ (b). Different symbols correspond to different times (filled symbols for the real part, empty symbols for the imaginary part). The lines are the real (full lines) and imaginary (dashed lines) part of the analytic correlation function in the non-equilibrium steady-sate given by Eq. (44). (c) The behavior of the imaginary part of the correlation function evaluated for different rescaled times $t / L$. As expected, if $t \ll L$, the numerically evaluated correlation function agrees with the NESS prediction in (47). Otherwise, for larger rescaled time $t / L \gg 1$ the correlation function takes the shape of two traveling peaks with velocity $w=2 \pi / L$. For the sake of clarity the curves are vertically shifted by an amount $\delta_{\text {shift }}=0.04(t / L)$. Notice the perfect agreement with peak's law of motion $x_{\text {peak }}(t / L)=2 \pi(t / L)$.

then a completely new phenomenon appears (see Figure 6 (c)): the imaginary part of the correlation function takes the shape of two traveling peaks which move with velocity $w=2 \pi / L$. The proper rescaled time in order to take trace of such a behavior is $t / L$ : in terms of this "new time", the peaks propagate in space with a "rescaled velocity" $\tilde{w}=2 \pi$ which remains finite. In the region between these two moving peaks the imaginary part of the correlation function vanishes a part from small fluctuations due to finite size effects. This behavior is reminiscent of what was already observed in Ref. 30 and shows the crucial role of the boundaries for the system equilibration. In the following we analyze in details this equilibration regime.

At large times, $t \gg L$ (with $t / L^{2} \ll 1$ to avoid revivals), the free boundaries of the system matter for the properties of the correlation function $C(x, y)$ in the vicinity of the connection point. In this limit, in the double sum of Eq.(27) only the diagonal terms $m=n$ survive $^{71}$. Therefore, the large-time limit of the correlation function in a system with boundaries reduces to just the real contribution $^{72}$.

$$
C_{\infty}(x, y)=\sum_{n=0}^{\infty} \varphi_{p_{n}}(x) \varphi_{p_{n}}(y)\left\langle\hat{n}_{p_{n}}\right\rangle_{0} \equiv C_{R e}^{S t a t}(x-y)
$$

In other words, the role of the boundaries is to elastically reflect the particles. Moreover, particles with different momenta will reach the edges at different times causing a global dephasing which increases with time ${ }^{22,30}$. In this regime, at large rescaled times $t / L$, any current will be suppressed which is reflected in the vanishing of the imaginary part of the correlation function as seen in Figure 6 (c). We further support this picture by means of a numerical analysis showed in Figure $7(\mathrm{a}, \mathrm{b})$ where we plot the absolute value of the imaginary part of the correlation function for large rescaled times and two different initial conditions, i.e. $\beta_{\mathcal{L}}=1, \beta_{\mathcal{R}}=0.5$ and $\beta_{\mathcal{L}}=1, \beta_{\mathcal{R}}=5$. It is evident from this figure that, for sufficiently large rescaled times $t / L$, the imaginary part of the two-point function vanishes a part from small fluctuations which are suppressed as the system size $L$ increases.

In order to make this qualitative behavior more quantitate, we introduce a measure of these fluctuations by integrating the absolute value of $\operatorname{Im}[C(x, 0 ; t)]$ in a local domain $[-\Delta / 2, \Delta / 2] \subset[-L / 2, L / 2]$,

$$
\delta C_{\Delta}(L, t) \equiv \int_{-\Delta / 2}^{\Delta / 2} d x|\operatorname{Im}[C(x, 0 ; t)]|
$$

Numerical evidences reported in Figure 7 (c,d) show that for $1 \ll t / L \ll L$ the absolute deviation $\delta C_{\Delta}(L, t) \lesssim$ $\varepsilon_{\Delta}(L)$ is bounded where the bound $\varepsilon_{\Delta}(L)$ shows a numerical scaling $\sim \Delta / L$. As a consequence, if the local domain $\Delta$ is scaled proportionally to the system size, the perfect equilibration toward Eq. (51) will be never seen. This shows that equilibrium, where the correlators are given by (51), is reached only in a local sense, that is when the size $\Delta$ of the domain considered around the origin is such that $\Delta(L) \sim o(L)$.

Finally, another interesting feature evident from Figure 7 (d) is that the time $t$ required for the correlation function to reach a stationary value in the local domain $[-\Delta / 2, \Delta / 2]$ increases with $\Delta$, as it should be. Indeed, remember that the peaks in $\operatorname{Im}[C(x, y ; t)]$ move with a velocity $w=2 \pi / L$. For that reason, the larger is the 

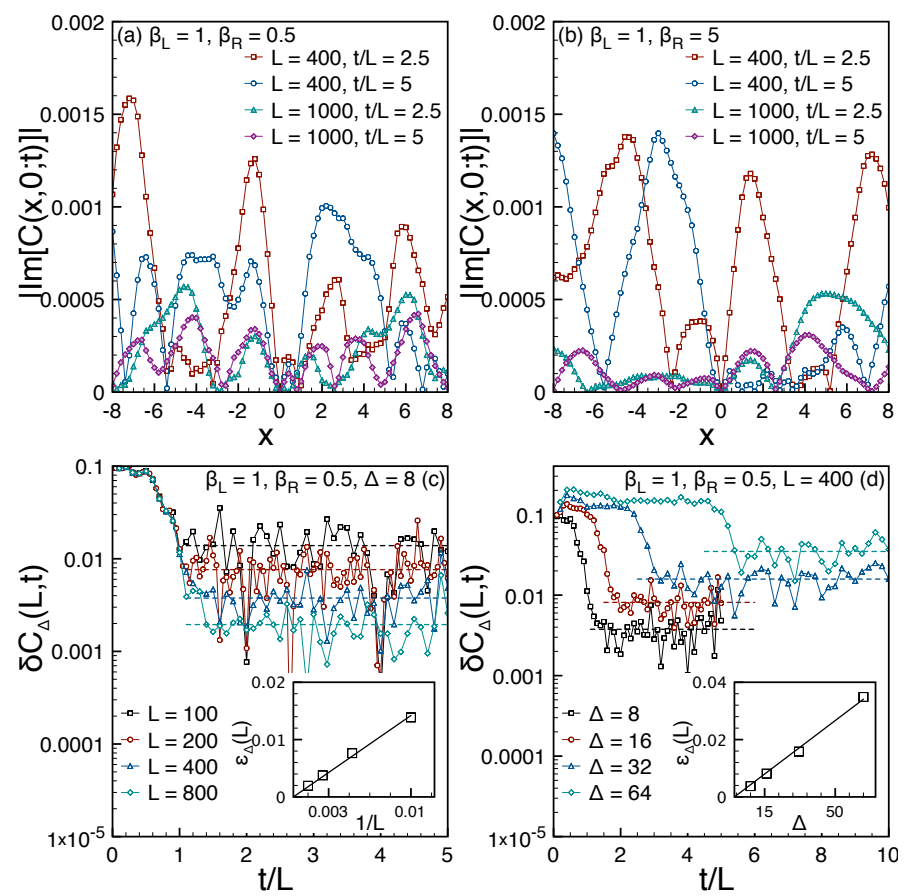

Figure 7: $(\mathrm{a}, \mathrm{b})$ The absolute value of the imaginary part fo the correlation function numerically evaluated for different $L$ and $t / L$. At fixed $t / L \gg 1$, we expect that $\operatorname{Im} C(x, y ; t) \rightarrow 0$ for $L \rightarrow \infty$. As a support of this, notice how for the times reported in figure, the fluctuations are $\lesssim 0.0015$ for $L=400$ and for $L=1000$ they become $\lesssim 0.0005$ giving a numerical evidence that $\operatorname{Im}[C(x, y ; t)]$ is approaching zero. (c,d) Integrated imaginary fluctuations $\delta C_{\Delta}(L, t)$ as a function of the rescaled time $t / L$ for: different sizes $L$ and fixed $\Delta=8$ (c); fixed $L=400$ and different subdomains $\Delta(\mathrm{d})$. The dashed lines represent the qusi-bounds $\varepsilon_{\Delta}(L)$ defined in the main text. Finally, in the insets of $(\mathrm{c}, \mathrm{d})$ we show the scaling of $\varepsilon_{\Delta}(L)$ respectively vs $1 / L$ or vs $\Delta$.

local domain wherein one analyzes the correlation function, the larger is the time one has to wait in order to expel out the peaks and see the stationary behavior.

Summarizing, once $\Delta$ is fixed, we can individuate four regimes in the time-evolution:

i) an initial transient during which the correlation function is not yet translational invariant and it is approaching the non-equilibrium steady-state;

ii) a NESS regime, for $t \gg 1$ but $t / L \ll 1$, corresponding locally to the non-equilibrium current-carrying state, wherein the correlators are given by Eq. (44);

iii) an intermediate regime whose duration extends up to the rescaled time $(t / L)^{*} \simeq \Delta /(2 \tilde{w})$ and which correspond to the intermediate almost constant plateaus in Figure 7 (d): in this regime the nonequilibrium correlators (namely Eq.(47)) are already destroyed but the moving peaks are still inside the region $[-\Delta / 2, \Delta / 2]$;

iv) finally, the equilibrium stationary state for $t \gg L$, with no more currents and characterized by the correlation function in Eq. (51).

Notice that, the first two regimes refer to the behavior of the correlation function reported in Fig. $7(\mathrm{c}, \mathrm{d})$ for $t / L \sim$ 0 and, therefore, are suppressed in that figure. Once again, let us emphasize that the regime iv) will be never reached if $\Delta \sim L$ or if we remove the boundary effects from the very beginning of the calculation. Naïvely, the last condition simply means that if we consider $L \rightarrow \infty$ from the very beginning then $t / L \ll 1$ for any finite $t$ and only the regimes i-ii) will survive.

\section{THE REDUCED DENSITY MATRIX AND STATISTICAL ENSEMBLES}

\section{A. Generalized Gibbs Ensemble}

In the previous section we have found the analytical form of the stationary two-point correlation function in the large-time limit. We have seen that, whenever a proper time-space scaling is done, in order to retain the effects of the boundaries, this function depends only on the post-quench mode occupation $n(p)$, and thanks to the Wick's theorem, all other observables can be described in terms of $n(p)$. With some abuse of language, we can say that the stationary state of the system after the quench can be described by the so called Generalized Gibbs Ensemble $(\text { GGE })^{13-16}$

$$
\hat{\rho}_{G G E}=Z_{G G E}^{-1} \exp \left\{-\sum_{j} \gamma_{j} \hat{I}_{j}\right\} \equiv Z_{G G E}^{-1} \mathrm{e}^{-\hat{H}_{e f f}}
$$

where $\hat{I}_{j}$ are local integral of motion, $\operatorname{Tr} \hat{\rho}_{\mathrm{GGE}}=1$, and where we defined an "effective" Hamiltonian $\hat{H}_{\text {eff }}$. The Lagrange multipliers $\gamma_{j}$ are fixed by the initial state trough the conditions $\operatorname{Tr}\left[\hat{\mathrm{I}}_{\mathrm{j}} \hat{\rho}_{\mathrm{GGE}}\right]=\left\langle\hat{\mathrm{I}}_{\mathrm{j}}\right\rangle_{0}$. However, whenever a closed system evolves unitarily, even if the initial state is not prepared in a pure state, to talk about a stationary state $\hat{\rho}_{G G E}$ describing the whole system may seem paradoxical. This paradox is resolved using the reduced density matrix $\hat{\rho}_{A}(t) \equiv$ $\operatorname{Tr}_{\mathrm{B}}[\hat{\rho}(\mathrm{t})]$, where $B$ is the complement of $A$ and $\hat{\rho}(t)=$ $\exp (-i \hat{H} t) \hat{\rho}_{0} \exp (i \hat{H} t)$ is the time-evolved density matrix of the whole system ${ }^{15,21,22,24,25,66}$. Indeed, we should think at the GGE in a "local" sense saying that the system reaches a stationary state if, after properly taking the TD limit, the limit $\hat{\rho}_{A, \infty} \equiv \lim _{t \rightarrow \infty} \hat{\rho}_{A}(t)$ exists for any finite $A$. Then, we say that it is described by a statistical ensemble $\hat{\rho}_{E}$ if the reduced density matrix $\hat{\rho}_{A, E} \equiv \operatorname{Tr}_{\mathrm{B}}\left[\hat{\rho}_{\mathrm{E}}\right]$ equals $\hat{\rho}_{A, \infty}$. In practice, carrying out the time evolution of the reduced density matrix is not a trivial task. However, for a fermionic quadratic theory, it has been shown that the reduced density matrix can be written as an exponential of the fermionic fields ${ }^{67-69}$

$$
\hat{\rho}_{A}=Z_{A}^{-1} \exp \left\{-\int_{A} d x d y \hat{\Psi}^{\dagger}(x) S_{A}(x, y ; t) \hat{\Psi}(y)\right\},
$$


where the function $S_{A}(x, y ; t)$ is connected to the restriction over the region $A$ of the correlation matrix $C(x, y ; t) \equiv\left\langle\hat{\Psi}^{\dagger}(x) \hat{\Psi}(y)\right\rangle_{t}$ via the formal relation

$$
S_{A}(x, y ; t)=\ln \frac{\delta_{A}(x-y)-C_{A}(x, y ; t)}{C_{A}(x, y ; t)},
$$

where $C_{A}(x, y ; t)=C(x, y ; t), \forall x, y \in A$, and $\delta_{A}(x-y)$ is the Dirac Delta function restricted in $A$. Thanks to this fact, if the two-point correlation function at large times is described by a statistical ensemble, then the expectation value of any local observable will also be.

For free fermion models, instead of using the local charges $\hat{I}_{j}$, we can work with the post-quench occupation modes $\hat{n}_{p_{n}}$, which are linearly connected with the local charges $^{29,30,70}$. Therefore, in the quench from a tensor thermal state in a non-interacting fermionic theory, the GGE will be

$$
\begin{aligned}
\hat{\rho}_{G G E} & =Z_{G G E}^{-1} \exp \left\{-\sum_{n=0}^{\infty} \lambda_{p_{n}} \hat{n}_{p_{n}}\right\}, \\
& =Z_{G G E}^{-1} \exp \left\{-L \int_{0}^{\infty} \frac{d p}{\pi} \lambda(p) \hat{n}(p)\right\},
\end{aligned}
$$

where the lagrange multipliers are determined via $[1+$ $\left.\mathrm{e}^{\lambda(p)}\right]^{-1}=n(p)$. In Figure 8 we plot the GGE correlation function $C_{G G E}(x, y) \equiv \operatorname{Tr}\left[\hat{\Psi}^{\dagger}(x) \hat{\Psi}(y) \hat{\rho}_{G G E}\right]=C_{\infty}(x, y)$, for some initial conditions.

It is worth mentioning that, expanding $\lambda(p)$ around zero and using the parity of $n(p)$, one can rewrite the Eq. (56) in terms of the local charges

$$
\begin{aligned}
\hat{I}_{2 j} & \equiv(L / \pi) \int_{0}^{\infty} d p p^{2 j} \hat{n}(p) \\
& =\iint d x d y(-1)^{j} \hat{\Psi}^{\dagger}(x) \delta^{(2 j)}(x-y) \hat{\Psi}(y) \\
& =\int d x \hat{\Psi}^{\dagger}(x)(-1)^{j} \partial_{x}^{2 j} \hat{\Psi}(x)
\end{aligned}
$$

with Lagrange multipliers $\gamma_{2 j}=\left(\left.\partial_{p}^{2 j} \lambda(p)\right|_{0}\right) /(2 j)$ !, obtaining for the "effective" Hamiltonian the following expression

$$
\begin{aligned}
\hat{H}_{e f f} & =\sum_{j} \gamma_{2 j} \hat{I}_{2 j} \\
& =\int d x \hat{\Psi}^{\dagger}(x)\left[\sum_{j}(-1)^{j} \gamma_{2 j} \partial_{x}^{2 j}\right] \hat{\Psi}(x) .
\end{aligned}
$$

Now some comments are due. As it should, $\hat{H}_{e f f}$ is local in the sense that it is an integral of a local current. Nevertheless, the differential operator $\sum_{j}(-1)^{j} \gamma_{2 j} \partial_{x}^{2 j}$ acting on the local fields contains all the even derivatives: in other words, depending on the behavior of $\gamma_{2 j}$, the "effective" interaction could be long-range.

It is straightforward to show that $\gamma_{0}=0$ and $\gamma_{2}=$ $\left(\beta_{\mathcal{L}}+\beta_{\mathcal{R}}\right) / 2$. This result states that, in the asymptotic stationary state, the Lagrange multiplier associated to

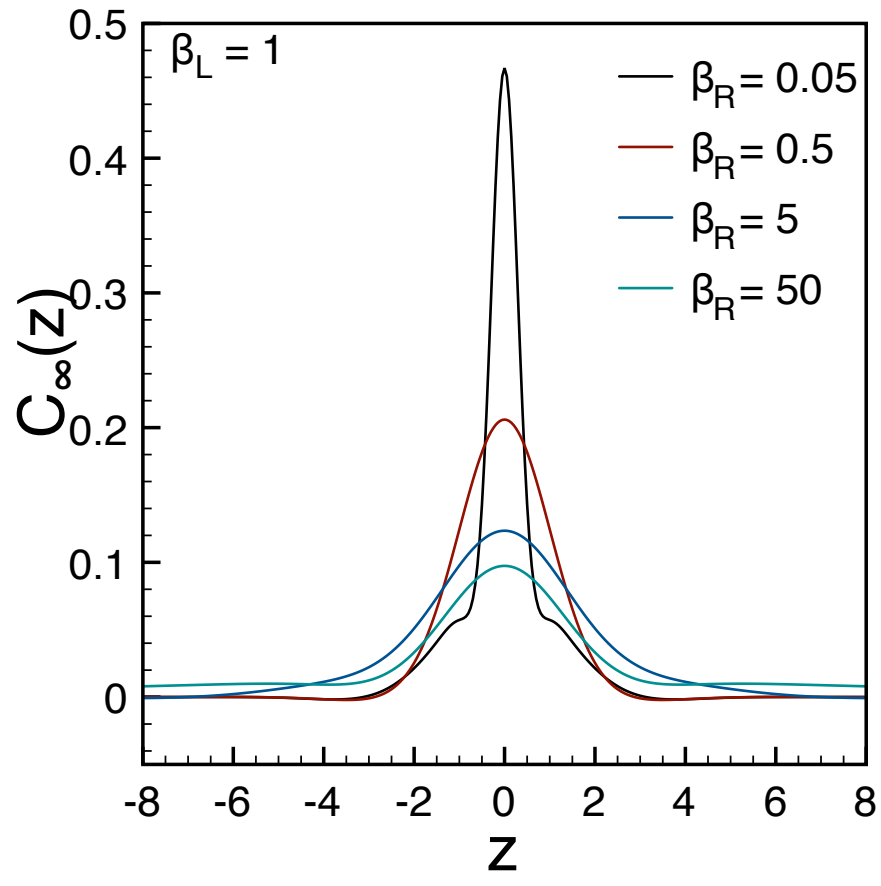

Figure 8: Stationary correlation function $C_{\infty}(z)$, which agrees with the GGE correlation function, for a system with boundaries initially prepared with $\beta_{\mathcal{L}}=1$ and $\beta_{\mathcal{R}}=0.05,0.5,5,50$.

the post-quench Hamiltonian $\left(\hat{H} \equiv \hat{I}_{2}\right)$, i.e. the "equilibrium inverse temperature", equals the average of the initial inverse temperatures. This particular result is an effect of the coexistence of the entire set of conserved charges and, as we will see in the next paragraph, it will not survive if one considers a truncated set of local charges. Moreover, it can be easily shown that $\gamma_{2 j}$ decays exponentially for large $j$ making the "effective" Hamiltonian $\hat{H}_{\text {eff }}$ short-range interacting, in contrast to what was observed in Ref. 43 for the non-equilibrium stationary state following a similar quench in the XX model, where it was found that the effective Hamiltonian is longrange interacting. ${ }^{73}$

Once again, we want to stress that the description of the large-time stationary-state via the GGE is correct only if the effect of the boundaries is retained in the TD limit. In other words, the proper scaling which permits to see a true equilibrium state (without any current) is obtained only by considering $L \rightarrow \infty$ with $t / L \gg 1$ and $t / L^{2} \ll 1$.

\section{B. Comparing the GGE with the grand canonical ensemble}

A generic non-integrable system, with conserved number of particles, admits only $\hat{N}$ and $\hat{H}$ as local conserved charges. Therefore, if a large-time stationary state exists it should be described by the Grand Canonical Ensemble 

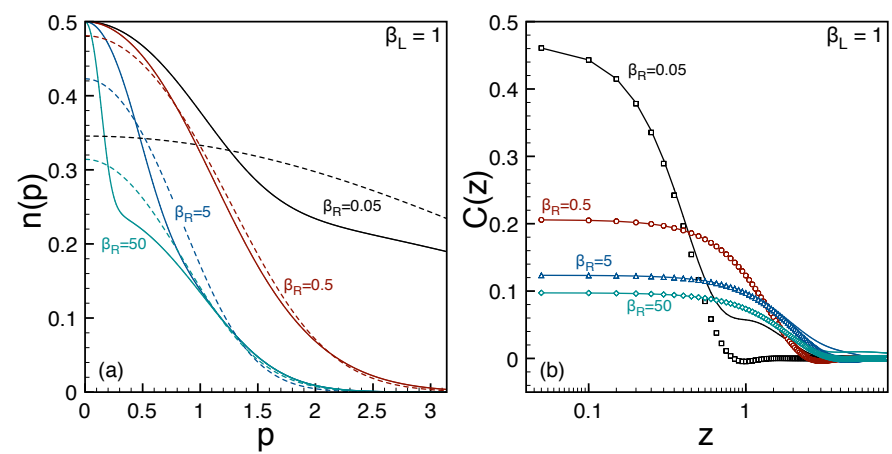

Figure 9: (a) Post-quench mode occupation in the GGE (full lines) and in the GCE (dashed lines) evaluated for different initial temperatures $\beta_{\mathcal{L}}=1$ and $\beta_{\mathcal{R}}=0.05,0.5,5,50$. (b) Two-point correlation function in the GGE (full lines) and in the GCE (symbols).

$(\mathrm{GCE})$

$$
\hat{\rho}_{G C E}=Z_{G C E}^{-1} \mathrm{e}^{-\mu_{G C} \hat{N}-\beta_{G C} \hat{H}},
$$

where, once again, the chemical potential $\mu_{G C}$ and the inverse temperature $\beta_{G C}$ are fixed by the initial conditions $\langle\hat{N}\rangle_{0}=\operatorname{Tr}\left[\hat{\rho}_{G C E} \hat{N}\right],\langle\hat{H}\rangle_{0}=\operatorname{Tr}\left[\hat{\rho}_{G C E} \hat{H}\right]$.

It is worth investigating the qualitative and quantitative differences between the grand canonical ensemble and the GGE to provide testable predictions for experiments and numerical analyses. In our case, in order to estimate the differences in the expectation values of local observables, we compare the results previously obtained in the GGE with the grand canonical ensemble. In particular, the two Lagrange multipliers $\mu_{G C}$ and $\beta_{G C}$ are fixed by the following set of equations (already in the TD limit)

$$
\begin{aligned}
\int_{0}^{\infty} \frac{d p}{\pi} \frac{1}{1+\mathrm{e}^{\beta_{G C} p^{2}+\mu_{G C}}} & =\frac{n_{\mathcal{L}}+n_{\mathcal{R}}}{2} \\
\int_{0}^{\infty} \frac{d p}{\pi} \frac{p^{2}}{1+\mathrm{e}^{\beta_{G C} p^{2}+\mu_{G C}}} & =\frac{\mathcal{E}_{\mathcal{L}}+\mathcal{E}_{\mathcal{R}}}{2}
\end{aligned}
$$

which can be rewritten in terms of polylogarithm functions $\operatorname{Li}_{n}(z)=\sum_{k=1}^{\infty} z^{k} / k^{n}$ as

$$
\begin{aligned}
& \operatorname{Li}_{1 / 2}\left(-\mathrm{e}^{-\mu_{G C}}\right)=-\beta_{G C}^{1 / 2} \sqrt{\pi}\left(n_{\mathcal{L}}+n_{\mathcal{R}}\right) \\
& \operatorname{Li}_{3 / 2}\left(-\mathrm{e}^{-\mu_{G C}}\right)=-2 \beta_{G C}^{3 / 2} \sqrt{\pi}\left(\mathcal{E}_{\mathcal{L}}+\mathcal{E}_{\mathcal{R}}\right),
\end{aligned}
$$

from which $\mu_{G C}$ is given by the solution of

$$
\frac{\mathrm{Li}_{3 / 2}\left(-\mathrm{e}^{-\mu_{G C}}\right)}{\left[\mathrm{Li}_{1 / 2}\left(-\mathrm{e}^{-\mu_{G C}}\right)\right]^{3}}=\frac{2}{\pi} \frac{\mathcal{E}_{\mathcal{L}}+\mathcal{E}_{\mathcal{R}}}{\left(n_{\mathcal{L}}+n_{\mathcal{R}}\right)^{3}}
$$

and, therefore, $\beta_{G C}$ is obtained plugging the numerically found $\mu_{G C}$ in one of the two equations in Eq.(65). In Figure 9 (a) we compare the GGE mode occupation distribution with the grand canonical results. Notice how the GCE gives completely different predictions with respect to the exact GGE results. In particular, the discrepancy reduces as the two initial temperatures approach to each other. Actually, for $\beta_{\mathcal{L}}=\beta_{\mathcal{R}}$, the initial state is already a thermal state and, in this case, the dynamics is trivial.

Nonetheless, as far as short-range correlation function is concerned, the grand canonical prediction coincides with the GGE prediction up to order $O\left(z^{4}\right)$. Indeed, expanding $C_{G G E}(z)$ around $z=0$ one has

$$
\begin{aligned}
C_{G G E}(z) & =\int_{0}^{\infty} \frac{d p}{\pi} n(p)-\frac{z^{2}}{2} \int_{0}^{\infty} \frac{d p}{\pi} p^{2} n(p)+O\left(z^{4}\right) \\
& =\frac{n_{\mathcal{L}}+n_{\mathcal{R}}}{2}-\frac{\mathcal{E}_{\mathcal{L}}+\mathcal{E}_{\mathcal{R}}}{2} \frac{z^{2}}{2}+O\left(z^{4}\right)
\end{aligned}
$$

which agrees with the $2^{\text {nd }}$-order expansion of the correlation function evaluated in the GCE. In Figure 9 (b) we compare, in log-scale, the GGE and the GCE correlation function.

\section{CONLUSIONS}

In this paper we studied analytically and numerically the non-equilibrium dynamics of a Fermi gas initially prepared into two halves at different temperatures. After putting in contact the two halves, the system is left to evolve with a non-interacting Hamiltonian. In a first step, we considered an infinitely extend system and we fully characterized the dynamics of the particles and energy profiles by means of a hydrodynamic approach ${ }^{59-61}$. From those results we obtained the analytical expression for the particle and energy currents which perfectly matches the CFT predictions ${ }^{62}$.

Nevertheless, we argued that, the non-equilibrium stationary state describing such currents represents a regime which is completely destroyed whenever the system is finite. Real systems are usually finite indeed, and the boundaries should play a crucial role in the equilibration mechanism.

Thus, we stressed that the mechanism which leads to the equilibration is due to the interference of the particles going around the finite system many times ${ }^{30}$. We prove that for long time and in a proper TD limit, i.e. taking into account the effects of the boundaries, any finite subsystem becomes truly stationary and its behavior is described by a GGE which only depends on the postquench occupation mode distribution. This provides a proof of a GGE for an inhomogeneous initial state constructed from two halves at two different temperatures.

\section{ACKNOWLEDGEMENTS}

The authors are grateful to Viktor Eisler and Giuseppe Mussardo for correspondence. M. C. thanks Pasquale Calabrese for helpful discussions and acknowledges the ERC for financial support under Starting Grant 279391 EDEQS. 
1 M. Greiner, O. Mandel, T. W. Hänsch, and I. Bloch, Nature 41951 (2002).

2 T. Kinoshita, T. Wenger, D. S. Weiss, Nature 440, 900 (2006).

3 S. Hofferberth, I. Lesanovsky, B. Fischer, T. Schumm, and J. Schmiedmayer, Nature 449, 324 (2007).

4 S. Trotzky Y.-A. Chen, A. Flesch, I. P. McCulloch, U. Schollwöck, J. Eisert, and I. Bloch, Nature Phys. 8, 325 (2012).

5 M. Cheneau, P. Barmettler, D. Poletti, M. Endres, P. Schauss, T. Fukuhara, C. Gross, I. Bloch, C. Kollath, and S. Kuhr, Nature 481, 484 (2012).

6 M. Gring, M. Kuhnert, T. Langen, T. Kitagawa, B. Rauer, M. Schreitl, I. Mazets, D. A. Smith, E. Demler, and J. Schmiedmayer, Science 337, 1318 (2012).

7 U. Schneider, L. Hackermüller, J. P. Ronzheimer, S. Will, S. Braun, T. Best, I. Bloch, E. Demler, S. Mandt, D. Rasch, and A. Rosch, Nature Phys. 8, 213 (2012).

8 J. P. Ronzheimer, M. Schreiber, S. Braun, S. S. Hodgman, S. Langer, I. P. McCulloch, F. Heidrich-Meisner, I. Bloch, and U. Schneider, Phys. Rev. Lett. 110, 205301 (2013).

9 M. J. Mark, E. Haller, K. Lauber, J. G. Danzl, A. Janisch, H. P. Büchler, A. J. Daley, and H.-C. Nägerl, Phys. Rev. Lett. 108, 215302 (2012).

10 T. Fukuhara, A. Kantian, M. Endres, M. Cheneau, P. Schauß, S. Hild, D. Bellem, U. Schollwöck, T. Giamarchi, C. Gross, I. Bloch, and S. Kuhr, Nature Physics 9, 235 (2013).

11 F. Meinert, M. J. Mark, E. Kirilov, K. Lauber, P. Weinmann, A. J. Daley, and H.-C. Nägerl, Phys. Rev. Lett. 111, 053003 (2013).

12 A. Polkovnikov, K. Sengupta, A. Silva, and M. Vengalattore, Rev. Mod. Phys. 83, 863 (2011).

13 M. Rigol, V. Dunjko, V. Yurovsky, and M. Olshanii, Phys. Rev. Lett. 98, 50405 (2007).

14 M. Rigol, V. Dunjko, and M. Olshanii, Nature 452, 854 (2008).

15 M. Rigol, Phys. Rev. Lett. 103, 100403 (2009); Phys. Rev. A 80, 053607 (2009).

16 M. Rigol and M. Srednicki, Phys. Rev. Lett. 108, 110601 (2012).

17 T. M. Wright, M. Rigol, M. J. Davis, K. V. Kheruntsyan, arXiv:1312.4657.

18 D. Fioretto and G. Mussardo New J. Phys. 12055015 (2010); S. Sotiriadis, D. Fioretto, G. Mussardo, J. Stat. Mech. P02017 (2012); G. Mussardo, Phys. Rev. Lett. 111, 100401 (2013); S. Sotiriadis, G. Takacs, G. Mussardo, Phys. Lett. B 734, 52 (2014).

19 P. Calabrese and J. Cardy, Phys. Rev. Lett. 96, 136801 (2006); J. Stat. Mech. P06008 (2007); J. Stat. Mech. P04010 (2005).

20 M. A. Cazalilla, Phys. Rev. Lett. 97, 156403 (2006); A. Iucci, and M. A. Cazalilla, Phys. Rev. A 80, 063619 (2009); New J. Phys. 12, 055019 (2010). M. A. Cazalilla,A. Iucci, and M.-C, Chung, Phys. Rev. E 85, 011133 (2012). A. Mitra and T. Giamarchi, Phys. Rev. Lett. 107, 150602 (2011).

21 M. Cramer, C. M. Dawson, J. Eisert, and T. J. Osborne, Phys. Rev. Lett. 100, 030602 (2008); M. Cramer and J. Eisert, New J. Phys. 12, 055020 (2010).

22 T. Barthel and U. Schollwöck, Phys. Rev. Lett. 100,
100601 (2008).

23 J.-S. Caux, F. Essler, Phys. Rev. Lett. 110257203 (2013).

24 P. Calabrese, F.H.L. Essler and M. Fagotti, Phys. Rev. Lett. 106, 227203 (2011); J. Stat. Mech. (2012) P07016; J. Stat. Mech. (2012) P07022.

25 S. Sotiriadis, P. Calabrese, and J. Cardy, EPL 87, 20002, (2009).

26 M. Fagotti, Phys. Rev. B 87, 165106 (2013).

27 F. H. L. Essler, S. Evangelisti, and M. Fagotti, Phys. Rev. Lett. 109, 247206 (2012).

28 D. Schuricht and F. H. L. Essler, J. Stat. Mech. (2012) P04017.

29 M. Fagotti and F. H. L. Essler, Phys. Rev. B 87, 245107 (2013).

30 M. Collura, S. Sotiriadis, and P. Calabrese, Phys. Rev. Lett. 110, 245301 (2013); J. Stat. Mech. P09025 (2013).

31 M. Kormos, M. Collura and P. Calabrese, Phys. Rev. A 89, 013609 (2014).

32 M. Fagotti, M. Collura, F. H. L. Essler and P. Calabrese, Phys. Rev. B 89, 125101 (2014).

33 L. Bucciantini, M. Kormos, P. Calabrese, J. Phys. A: Math. Theor. 47, 175002 (2014).

34 M. Fagotti, J. Stat. Mech. (2014) P03016.

35 C. J. Bolech, F. Heidrich-Meisner, S. Langer, I. P. McCulloch, G. Orso, and M. Rigol, Phys. Rev. Lett. 109, 110602 (2012).

36 C. Karrasch, J. Rentrop, D. Schuricht, and V. Meden, Phys. Rev. Lett. 109, 126406 (2012).

37 J. Rentrop, D. Schuricht and V. Meden, New J. Phys. 14, 075001 (2012).

38 A. Mitra, Phys. Rev. Lett. 109, 260601 (2012); Phys. Rev. B 87, 205109 (2013);

39 M. Tavora, and A. Mitra, Phys. Rev. B 88, 115144 (2013).

${ }^{40}$ L. Vidmar, S. Langer, I. P. McCulloch, U. Schneider, U. Schollwöck, and F. Heidrich-Meisner, Phys. Rev. B 88, 235117 (2013).

41 F.H.L. Essler, S. Kehrein, S.R. Manmana, N.J. Robinson, Phys. Rev. B 89, 165104 (2014).

${ }^{42}$ H. Araki and T. G. Ho, Proc. Steklov Inst. Math. 228, 191 (2000).

43 Y. Ogata, Phys. Rev E 66, 066123 (2002); Phys. Rev. E 66, 016135 (2002).

${ }^{44}$ W. H. Aschbacher and C.-A. Pillet, J. Stat. Phys. 112, 1153 (2003).

45 D. Karevski, Eur. Phys. J. B 27, 147 (2002); T. Platini, D. Karevski, Eur. Phys. J. B 48225 (2005); T. Platini, D. Karevski, J. Phys. A: Math. Theor. 40, 1711 (2007).

46 A. De Luca, J. Viti, D. Bernard and B Doyon, Phys. Rev. B 88, 134301 (2013).

47 C. Karrasch, R. Ilan and, J. E. Moore, Phys. Rev. B 88, 195129 (2013).

48 M. Mintchev, J. Phys. A 44, 415201 (2011).

49 T. Antal, Z. Rácz, A. Rákos, and G.M. Schütz, Phys. Rev. E 57, 5184 (1998).

50 D. Ruelle, J. Stat. Phys. 98, 57 (2000).

51 V. Jakšićand and C.-A. Pillet, Commun. Math. Phys. 226, 131 (2002).

52 D. Karevski, T. Platini, Phys. Rev. Lett. 102, 207207 (2009).

53 T. Prosen, Phys. Rev. Lett. 107, 137201 (2011).

54 D. Karevski, V. Popkov, G. M. Schuetz, Phys. Rev. Lett. 
110 047201(2013); V. Popkov, D. Karevski, G. M. Schuetz, Phys. Rev. E 88062118 (2013).

55 J. Lancaster, and A. Mitra, Phys. Rev. E 81, 061134 (2010).

56 T. Sabetta, and G. Minguich, Phys. Rev. B 88, 245114 (2013).

57 A. Zamolodchikov, Nucl. Phys. B 342, 695 (1990).

58 O. Castro-Alvaredo, Y. Chen, B. Doyon, M. Hoogeveen, J. Stat. Mech. (2014) P03011.

59 T. Antal, P. L. Krapivsky, and A. Rákos, Phys. Rev. E 78, 061115 (2008).

60 M. Collura, H. Aufderheide, G. Roux and D. Karevski, Phys. Rev. A 86, 013615 (2012).

61 P. Wendenbaum, M. Collura and D. Karevski, Phys. Rev. A 87, 023624 (2013).

62 D. Bernard and B. Doyon, J. Phys. A 45, 362001 (2012); arXiv:1302.3125. M.J. Bhaseen, B. Doyon, A. Lucas, K. Schalm, arXiv:1311.3655.

63 V. Eisler, Z. Zimboras, Phys. Rev. A 89, 032321 (2014).

${ }^{64}$ F. W. J. Olver, SIAM J. Math. Anal. 5, 19 (1974).

${ }^{65}$ R. Wong, Asymptotic Approximations of Integrals, Acad. Press, New York, (1989).

66 D. Rossini, A. Silva, G. Mussardo, and G. Santoro, Phys. Rev. Lett. 102, 127204 (2009); D. Rossini, S. Suzuki, G.
Mussardo, G. E. Santoro, and A. Silva, Phys. Rev. B 82, 144302 (2010).

67 P. Calabrese,M. Mintchev and E. Vicari, Phys. Rev. Lett. 107, 020601 (2011); J. Stat. Mech. P09028 (2011).

68 I. Peschel I, J. Phys. A: Math. Gen. 36, L205 2003; J. Stat. Mech. P06004 (2004); Braz. J. Phys. 42267 (2012).

69 I. Peschel and V. Eisler, J. Phys. A: Math. Gen. 42, 504003 (2009).

70 J.-S. Caux and R. M. Konik, Phys. Rev. Lett. 109, 175301 (2012).

71 Notice that the terms $m=-n$ are not allowed since $m$ and $n$ should be both nonnegative.

72 Notice that this result exactly coincides with the time average $\lim _{T \rightarrow \infty} T^{-1} \int_{0}^{T} d t C(x, y ; t)$

73 Indeed, in terms of the local conserved charges of the XXmodel, i.e. $\hat{Q}_{j}^{ \pm}=i^{(1 \mp 1) / 2} \sum_{i}\left(\hat{c}_{i}^{\dagger} \hat{c}_{i+j} \pm\right.$ h.c. $) / 2$, the effective Hamiltonian takes the form $\hat{H}_{e f f}=\hat{H}_{X X}+\sum_{j} \mu_{j}^{-} \hat{Q}_{j}^{-}$, with $\mu_{j}^{-} \sim 1 / j$ for large $j$, where $\hat{c}_{j}\left(\hat{c}_{j}^{\dagger}\right)$ are the Fermi annihilation (creation) operators on lattice and $\hat{H}_{X X}$ is the XX-model hamiltonian. However, we remand to Ref. 43 for further details. 